\title{
Dependency of the shell-less Culture on temperature and rotating velocity
}

\author{
Massimo Fioranelli ${ }^{1 *}$, Alireza Sepehri ${ }^{2 \dagger}$, Maria Grazia Roccia ${ }^{1}$ \\ ${ }^{1}$ Department of Nuclear Physics, sub-nuclear and radiation. G.Marconi University, Rome, Italy. \\ ${ }^{2}$ Research Institute for Astronomy and Astrophysics of Maragha (RIAAM), P.O. Box 55134-441, Maragha, Iran.
}

\begin{abstract}
Recently, some authors have developed a shell-less culture system for chick embryos by using a plastic film as culture vessels. We will show that velocity of rotation of vessels in incubator and temperature have direct relations with growth of chick embryos. We also obtain a mathematical relation between velocity, temperature and rate of growth of chick embryo.
\end{abstract}

Keywords:Temperature, Culture, Cell, Chick, Embryo, Rotation

\section{INTRODUCTION}

One of main subject in science is finding various methods for the shell-less culture of bird embryos with high hatchability. This field of science is helpful for the efficient generation of transgenic chickens, embryo manipulations, tissue engineering, and basic studies in regenerative medicine. Shelless cultrure involves culturing chick embryos with associated yolk and albumen outside of the eggshell and shell membranes. The technique allows direct access to and continuous observation of cultured embryos almost to the time of hatching. The plastic wrap/culture tripod technique which described for the first time in [1] allows normal embryonic growth and differentiation from 48 hours (in ovo preincubation age) through at least 10 days of total incubation. On the other hand, some studies have indicated that the chorioallantoic membrane (CAM) of chick embryos cultured under shell-less conditions is defective in calcium transport, probably as a result of the expression of an inactive form of the CaBP [2]. Also, some authors have developed a simple shell-less chick embryo culture system to study glucose-induced malformations. This system involves the culturing of chick embryos from the second day to the fifth day of incubation, with associated yolk and thick and thin albumen outside the egg shell. The glucose-induced malformations were found to be concentration- and stage-dependent, thus emphasizing the roles of the degree of hyperglycemia and the stage of embryonic development in diabetic growth anomalies [3]. In another research, a chick embryo shell-less culture system has been established and used of it to observe change in behavior caused by nicotine and substances from cigarette smoke [4]. And finally, some authors have suggested a shell-less culture system for chick embryos by using a plastic film as culture vessels. They have examined the conditions required for embryonic hatching by comparing factors such as the addition of calcium lactate and the presence or absence of an oxygen supply [5]. Motivated by these researches, we consider the effect of temperature and rotating velocity of vessels on the growth of cells in shell-less culture. We will show that there is a direct relation between temperature, rotating velocity and growth of cells.

The outline of the paper is as follows. In section II, we will consider the effect of temperature and rotating velocity of vessels on the growth of cells in shell-less culture by doing some experimental analyses. In section III, we will show that there is a direct mathematical relation between temperature, rotating velocity and growth of cells. The last section is devoted to conclusion.

\section{THE EFFECT OF TEMPERATURE AND ROTATING VELOCITY OF VESSELS ON THE GROWTH OF CELLS IN SHELL-LESS CULTURE: EXPERIMENTAL ANALYSES.}

\section{A:Materials and Methods}

Chicken Eggs

All of the fertilized eggs used in this consideration were Dekalb Brown eggs, which were obtained from a village in Neyshabur ( an small region in Iran).

\section{Culture Vessels}

Our culture vessels are the same used in [5], however type of incubating, temperature and rotation were different. Similar to [5], a $450 \mathrm{ml}$ polystylene plastic cup was applied as the pod for the culture vessel. A 1-1.5 cm diameter hole was made in the side of the cup approximately $2 \mathrm{~cm}$ from the bottom, and the hole was plugged with a cotton pledget as a filter. A $2 \mathrm{~mm}$ diameter plastic tube was inserted through the space between the pledget and the hole to provide an oxygen supply. An aqueous solution $(40 \mathrm{ml})$ of benzalkonium chloride was then added to the cup. A

\footnotetext{
* massimo.fioranelli@gmail.com

$\dagger$ alireza.sepehri@uk.ac.ir, alireza.sepehri2017@gmail.com
} 
polymethylpentene film was formed into a concave shape, carefully avoiding wrinkles and installed as an artificial culture vessel in the pod. A polystylene plastic cover was placed on top of the culture vessel.

\section{Embryo Culture, Incubating, temperature and rotation}

Some of fertilized chicken eggs were not incubated before transferring to the culture vessels. Their eggshell was wiped and cracked and the whole egg contents were transferred to the culture vessel without pre-incubating period. The culture vessels were divided to 40 groups. Each group were maintained at an special temperature and humidity in an incubator and rotated with an special velocity. For example, one group were maintained at $38^{0} \mathrm{C}$ and rotated with 120clockwise twice a day. One another group were maintained at $37.5^{0} \mathrm{C}$ and rotated with $120 \mathrm{clockwise}$ fourth a day. Also, some others were maintained at $33^{\circ} \mathrm{C}$ and rotated with 120 clockwise eight times a day. Other groups were incubating at other temperatures and rotating velocities.

Some other fertilized eggs were incubated three days before transferring to the culture vessels. Then, their eggshell was wiped and cracked and the whole egg contents were transferred to the culture vessels. They were divided to 10 groups again. Each group were maintained at an special temperature and humidity in an incubator and rotated with an special velocity.

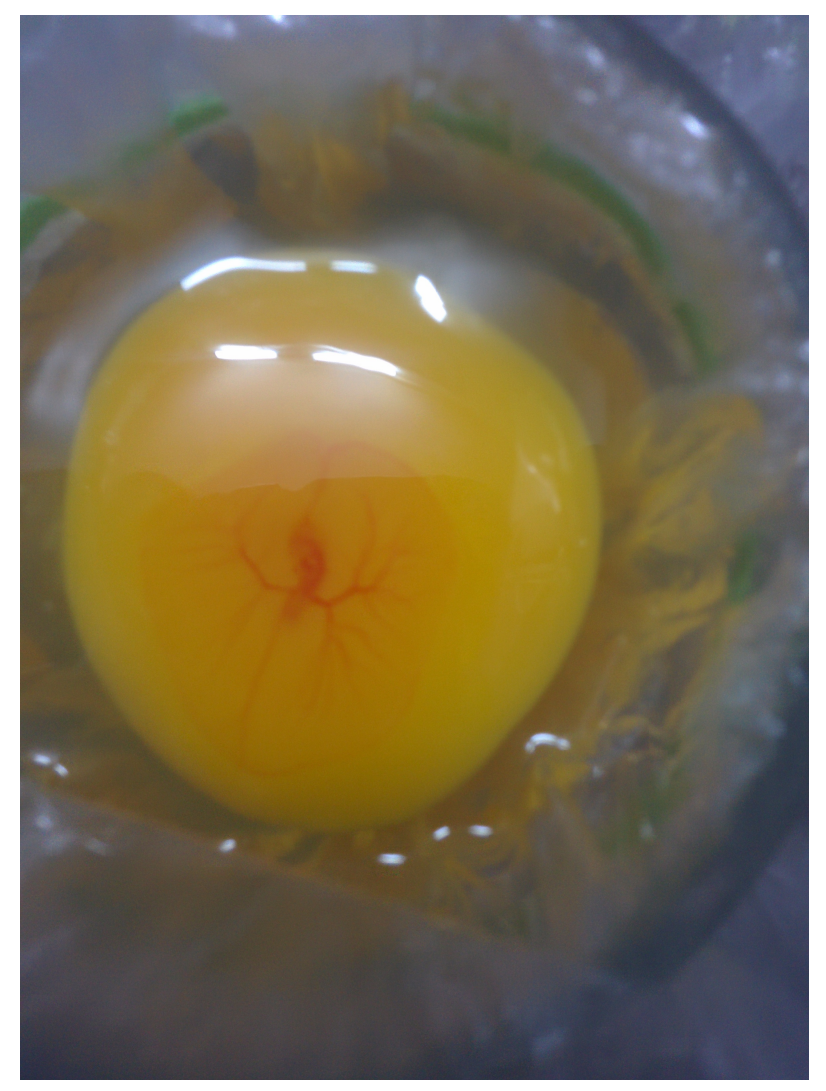

FIG. 1: Formation of chick embryo in shell-less culture vessel less than $48 \mathrm{~h}$ after incubating at $37.5^{0} C$.

\section{Results}

We show our results in figures (2-5). These figures are corresponded to shell-less cultures without passing preincubating stage. We observe that for some special temperatures and rotating velocities, shell-less chick embryos could be produced and survived more than 3 days. By decreasing temperature, chance of growing cells in shell-less cultures decreases. For increasing this probability, we have to increase the rotating velocity (See figure 2-4).

Our experiments indicate that there is a relation between temperature, rotating velocity and probability for producing chick embryo (See figure 5). In fact, there are some critical pints (certain temperatures and rotating velocities) that cells of chick embryo could grow better. Also, between these points $(T, \omega)$ there are some points that chick embryos die. For example, between $37.5^{\circ} \mathrm{C}$ and $38^{\circ} \mathrm{C}$, there are some temperatures like $37.8^{0} \mathrm{C}$ that growing of cells in shell-less culture becomes stop. 


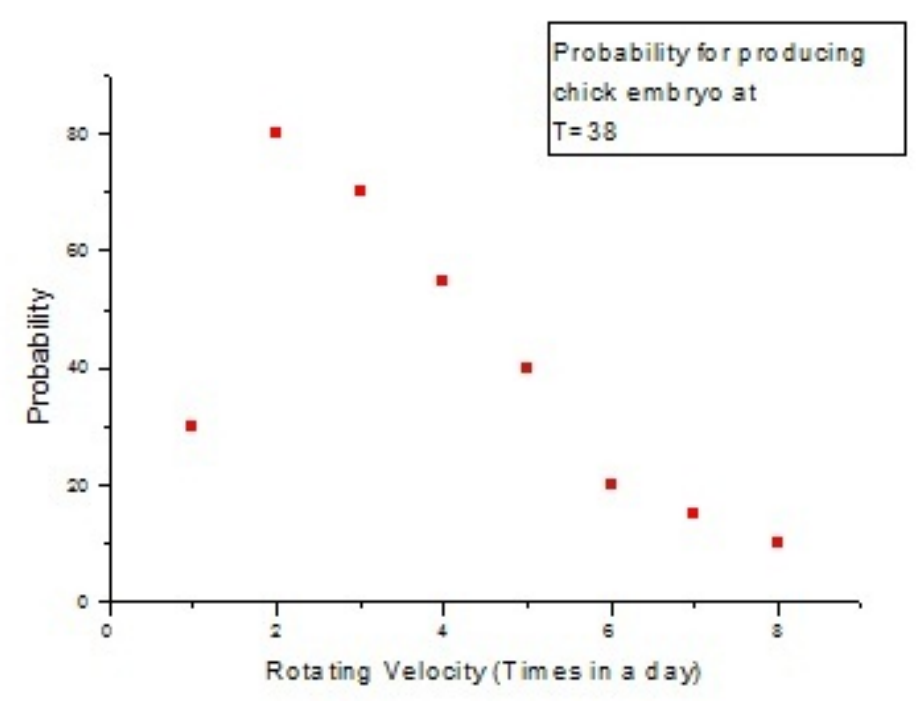

FIG. 2: Probability for producing chick embryo and surviving more than 3 days in terms of rotating velocity at $38^{0} C$.

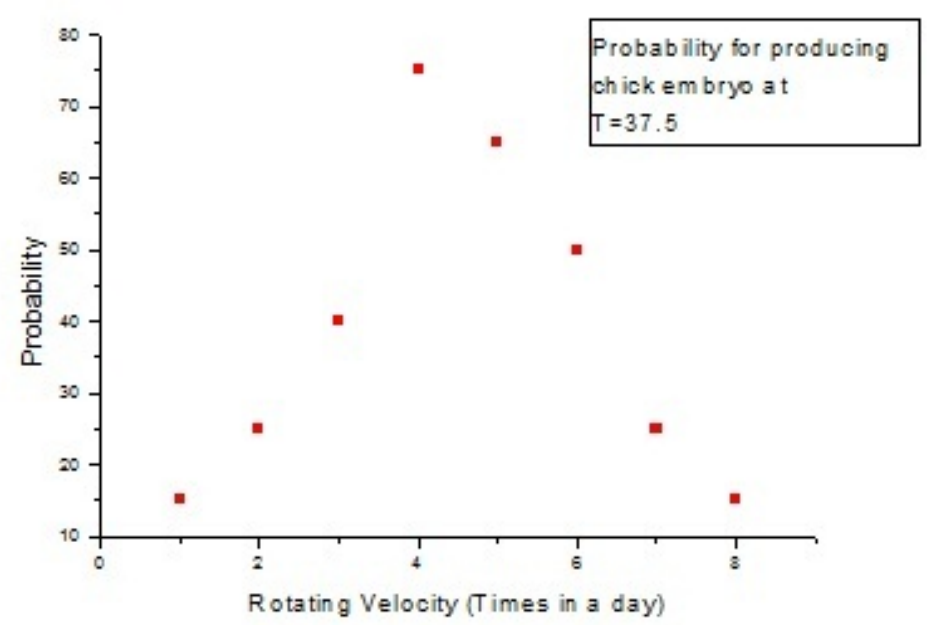

FIG. 3: Probability for producing chick embryo and surviving more than 3 days in terms of rotating velocity at $37.5^{0} C$.

\section{A MATHEMATICAL MODEL FOR GROWING CELLS IN SHELL-LESS CULTURE}

To obtain dependency of the rate of growing cells in shell-less culture system, we can use of entropy. Previously, it has been shown that entropy for rotating system can be obtained of below relation.

$$
S=\frac{4}{\pi T^{5}} \int_{r_{0}}^{\infty} d r \frac{F(r) r^{2}}{\sqrt{F^{2}(r)-F^{2}\left(r_{0}\right)}} \frac{4}{\cosh ^{4} \alpha}
$$

with new definition of $F(r)[6]$;

$$
F(r)=r^{2} \frac{4 \cosh ^{2} \alpha-3}{\cosh ^{4} \alpha}
$$




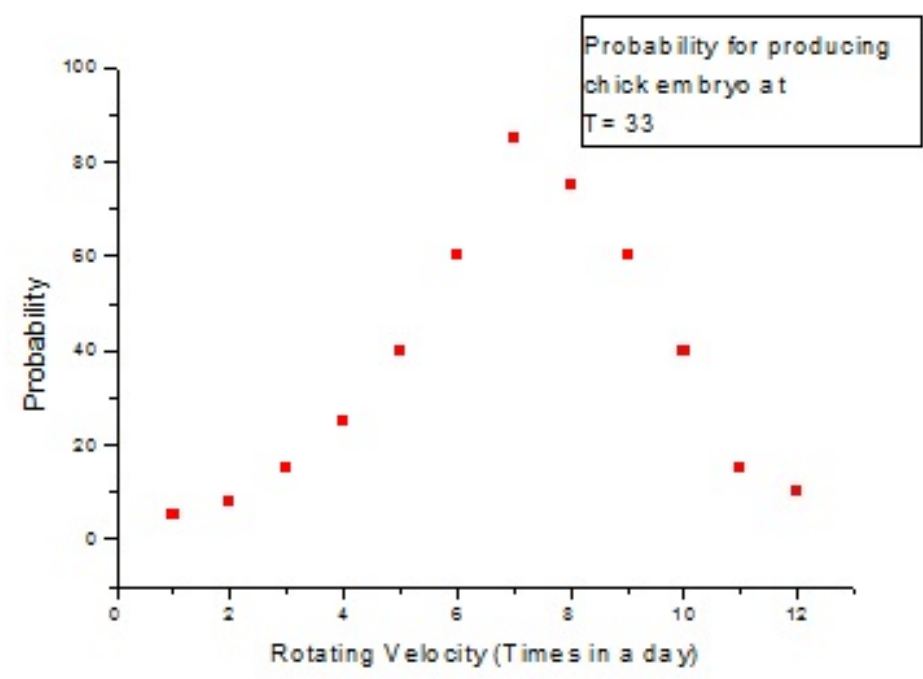

FIG. 4: Probability for producing chick embryo and surviving more than 3 days in terms of rotating velocity at $33^{\circ} C$.

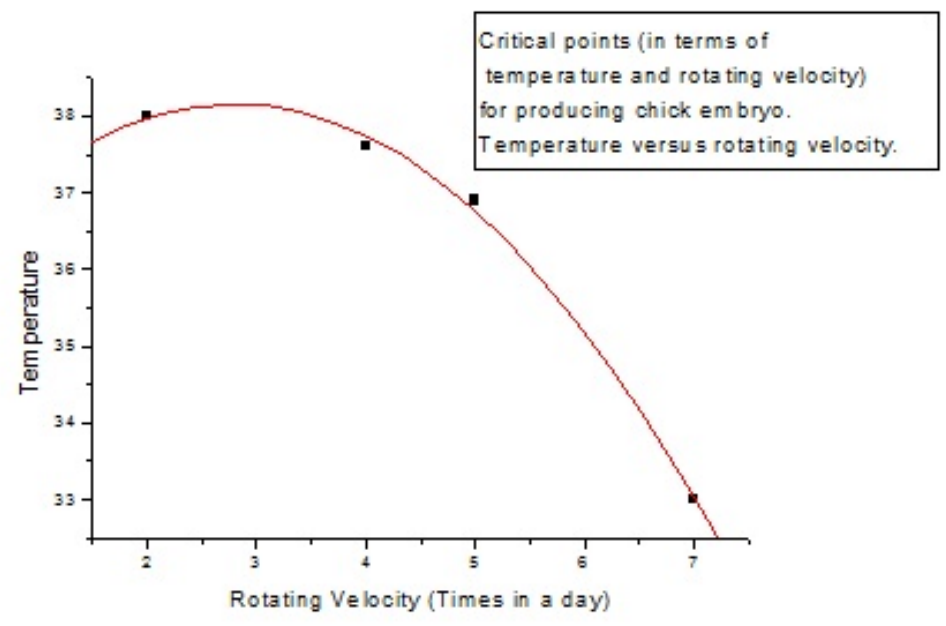

FIG. 5: Critical points (in terms of temperature and rotating velocity) for producing chick embryo. Temperature versus rotating velocity. $\left(T=A+B \omega+C \omega^{2}\right.$ with $\left.\mathrm{A}=35.86, \mathrm{~B}=1.63, \mathrm{C}=-0.29\right)$

and below definitions:

$$
\begin{aligned}
& \cosh ^{2} \alpha=\frac{3}{2} \frac{\cos \frac{\delta}{3}+\sqrt{3} \cos \frac{\delta}{3}}{\cos \delta} \\
& \cos \epsilon=\frac{1}{\sqrt{1+\frac{K^{2}}{r^{4}}}}
\end{aligned}
$$

And

$$
\cos \delta=\bar{T}^{4} \sqrt{1+\frac{K^{2}}{r^{4}}}
$$




$$
\bar{T}=\left(\frac{9 \pi^{2} N}{4 \sqrt{3}}\right)^{\frac{1}{2}} T
$$

where $S$ is the entropy of system, $r$ is the radius of rotation and $T$ is temperature. If shell-less culture vessels rotate with acceleration, we should write below relations between the coordinates of the accelerating system $(\tau, \sigma)$ and the coordinates of Minkowski space-time $(t, r)[7]$ :

$$
a t=e^{a \sigma} \sinh (a \tau) \quad a r=e^{a \sigma} \cosh (a \tau)
$$

On the other hand, it has been shown that this acceleration has a direct relation with temperature [7]:

$$
a \sim 2 \pi T
$$

In fact, by increasing temperature, the velocity of motion of each particle increases and each of them experience an type of acceleration. For this reason, a thermal system is equal to an accelerating system. On the other hand, temperature has a relation with angular velocity. thus, we can write:

$$
a \sim 2 \pi T=\frac{2 \pi T_{0}}{\sqrt{1-\frac{l_{0}^{2} \omega^{2}}{c^{2}}}}
$$

where $\omega$ is the angula velocity, $T_{0}$ is temperature in non-rotating system and $l_{0}$ is the radius of rotation in an accelerating frame. Using above equations, we can write below relations between the coordinates of the accelerating system $(\tau, \sigma)$ and the coordinates of Minkowski space-time $(t, r)$ :

$$
t=\frac{1}{\frac{2 \pi T_{0}}{\sqrt{1-\frac{l_{0}^{2} \omega^{2}}{c^{2}}}}} e^{a \sigma} \sinh \left(\frac{2 \pi T_{0}}{\sqrt{1-\frac{l_{0}^{2} \omega^{2}}{c^{2}}}} \tau\right) \quad r=\frac{1}{\frac{2 \pi T_{0}}{\sqrt{1-\frac{l_{0}^{2} \omega^{2}}{c^{2}}}}} e^{\frac{2 \pi T_{0}}{\sqrt{1-\frac{l_{0}^{2} \omega^{2}}{c^{2}}}}} \cosh (a \tau)
$$

Substituting above equation in equation (1), we obtain:

$$
\begin{aligned}
& S=\frac{4}{\pi T^{5}} \int_{l_{0}}^{\infty} d \sigma\left[\frac{1}{\frac{2 \pi T_{0}}{\sqrt{1-\frac{l_{0}^{2} \omega^{2}}{c^{2}}}}} e^{\frac{2 \pi T_{0}}{\sqrt{1-\frac{l_{0}^{2} \omega^{2}}{c^{2}}}}} \cosh (a \tau)\right]^{3} \times \\
& \frac{F\left(\frac{1}{\frac{2 \pi T_{0}}{\sqrt{1-\frac{l_{0}^{2} \omega^{2}}{c^{2}}}}} e^{\frac{2 \pi T_{0}}{\sqrt{1-\frac{l_{0}^{2} \omega^{2}}{c^{2}}}} \sigma} \cosh (a \tau)\right)}{\sqrt{F^{2}(r)-F^{2}\left(l_{0}\right)}} \frac{4}{\cosh ^{4} \alpha}
\end{aligned}
$$

Above entropy has a direct relation with number of cells that are created in shell-less culture system. By increasing temperatue or rotating velocity, this entropy increases and consequently, many cells are produced and chick embryo grows.

\section{SUMMARY AND DISCUSSION}

Recently, some authors have developed a shell-less culture system for chick embryos by using a plastic film as culture vessels. We have done similar experiments and obtained the same results. Then, we have changed the velocity of rotation of vessels in incubator and found tat there is a direct relation between velocity and growth of chick embryos. Next, we have repeated this experiments for different temperature and obtained a relation between temperature and rate of growth of chick embryo. Finally, we have proposed a mathematical model which allows us to obtain the relation between velocity, temperature and rate of growth of chick embryo in incubator. 


\section{Acknowledgements}

The work of A.Sepehri has been supported financially by the Research Institute for Astronomy and Astrophysics of Maragha (RIAAM),Iran under the Research Project NO.1/5237-79.

[1] Bruce E. Dunn, PhD Timothy P. Fitzharris, Bobby D. Barnett, The Anatomical Record, Volume 199, Issue 1, January 1981, Pages 33-43. https://doi.org/10.1002/ar.1091990105.

[2] Rocky S.Tuan, Developmental Biology, Volume 74, Issue 1, January 1980, Pages 196-204.https://doi.org/10.1016/00121606(80)90061-5.

[3] Savita Datar, Ramesh R. Bhonde, Rev Diabet Stud. 2005 Winter; 2(4): 221227. doi: 10.1900/RDS.2005.2.221.

[4] Shusei Hamamichi Hideo Nishigori, Toxicology Letters, Volume 119, Issue 2, 28 February 2001, Pages 95-102.

[5] Yutaka Tahara and Katsuya Obara, Journal of Poultry Science, 51 (3) pages 307-312(2014).

[6] Gianluca Grignani, Troels Harmark, Andrea Marini, Niels A. Obers, Marta Orselli, JHEP 1106:058,2011.

Gianluca Grignani, Troels Harmark, Andrea Marini, Niels A. Obers, Marta Orselli, Nucl.Phys.B851:462-480,2011.

[7] P.M. Alsing, I. Fuentes-Schuller, R.B. Mann, T.E. Tessier, Phys.Rev. A74 (2006) 032326. 